University of Nebraska - Lincoln

DigitalCommons@University of Nebraska - Lincoln

2009

Electron attachment to trans-azobenzene

Alberto Modelli

Paul Burrow

Follow this and additional works at: https://digitalcommons.unl.edu/physicsburrow

Part of the Atomic, Molecular and Optical Physics Commons

This Article is brought to you for free and open access by the Research Papers in Physics and Astronomy at DigitalCommons@University of Nebraska - Lincoln. It has been accepted for inclusion in Paul Burrow Publications by an authorized administrator of DigitalCommons@University of Nebraska - Lincoln. 


\title{
Electron attachment to trans-azobenzene
}

\author{
Alberto Modelli ${ }^{1,2}$ and Paul D. Burrow ${ }^{3}$ \\ 1 Dipartimento di Chimica “G. Ciamician”, Università di Bologna, via Selmi 2, \\ 40126 Bologna, Italy \\ 2 Centro Interdipartimentale di Ricerca in Scienze Ambientali (CIRSA), \\ Università di Bologna, via S. Alberto 163, 48123 Ravenna, Italy. Email \\ alberto.modelli@unibo.it; Fax: +39 051 2099456; Tel: +39 0512099522 \\ 3 Department of Physics and Astronomy, University of Nebraska-Lincoln, \\ Lincoln, NE 68588-0111, USA. Email pburrow1@unl.edu
}

\begin{abstract}
The temporary anion states of gas-phase trans-azobenzene are characterized by means of electron transmission spectroscopy (ETS) in the o- $6 \mathrm{eV}$ range. The measured energies of vertical electron attachment are compared with the energies of the $\pi^{*}$ virtual orbitals of the neutral molecule supplied by HF (at MP2 optimized geometries) and B3LYP calculations. The calculated energies, scaled with empirical equations, reproduce quantitatively the energies of the corresponding spectral features and predict a positive vertical electron affinity of $0.83 \mathrm{eV}$.

The total anion current at the walls of the collision chamber and the mass-selected molecular anion current are also reported as a function of the impact electron energy. In agreement with previous data, long-lived ( $>1 \mu \mathrm{s})$ parent molecular anions are detected at zero $\mathrm{eV}$ and near $1 \mathrm{eV}$. The close similarity of the electron transmission spectrum with the derivatives with respect to energy of the anion currents suggests strongly that shape resonances produced by electron capture into empty $\pi^{*}$ orbitals are the initial step in formation of the long lived molecular anions. This appears to rule out mechanisms in which direct formation of core-excited anion states are invoked. However, according to DFT calculations, conversion of the shape resonances around $1 \mathrm{eV}$ to longer-lived $\sigma-\pi^{*}$ core-excited doublet anion states is possible on energetic grounds.
\end{abstract}

Published in Physical Chemistry Chemical Physics 11 (2009), pp 8448-8455.

DOI: 10.1039/b9o8770b

Physical Chemistry Chemical Physics is copyright (C) 2009 by the Royal Society of

Chemistry. Used by permission.

Submitted 5 May 2009; accepted 12 June 2009; published 27 July 2009. 


\section{Introduction}

Azo compounds have been studied for over 70 years as chromophores employed in pigment and dye manufacturing. Yet since the first report by Hartley ${ }^{1}$ on its peculiar photochemical behavior, i.e., its ability to undergo reversible trans/cis isomerization upon light absorption, azobenzene continues to be the subject of new experimental and theoretical studies. ${ }^{2-12}$ Photo-isomerization occurs on the timescale of picoseconds, leading to drastic conformational and geometrical changes between the trans and (shorter) cis forms. This renders azobenzene oligomers and substituted azobenzenes the key components of photoswitched nanomechanical devices, suitable for a broad variety of applications in different fields (microelectronics, photoresponsive systems, optical lithography, liquid crystals, mechanical properties, optical devices, data storage, phase transitions, and biochemistry). The main challenge in the development and operation of these nano-machines is the need to interface them with the macroscopic world. A review of various applications has recently been reported. ${ }^{13}$

The electronic absorption spectra and the observed vibrational fine structure were studied ${ }^{14,15}$ in the gas phase and in solution. The spectrum of the most stable (by about $50 \mathrm{kjoule} / \mathrm{mol}^{16}$ ) trans isomer of azobenzene displays one symmetry forbidden $n(\sigma)-\pi^{*}$ transition around $450 \mathrm{~nm}(2.76 \mathrm{eV})$ and several symmetry allowed $\pi-\pi^{*}$ transitions at higher energy, the lowest one occurring at $329 \mathrm{~nm}(3.77 \mathrm{eV})$ in the gas phase. ${ }^{14}$ More recently it has been shown that azobenzenes can be manipulated with a scanning tunneling microscope tip when adsorbed on a gold surface, including rotation or translation ${ }^{17}$ and cis-trans isomerization, ${ }^{18}$ the latter process being still a subject of studies. ${ }^{19,20}$

The valence filled level structure of azobenzene in the gas phase was investigated ${ }^{21,22}$ by means of photoelectron spectroscopy. Electron ejection from the highest occupied molecular orbital (HOMO), assigned to a $\sigma \mathrm{MO}$ with mainly nitrogen lone pair character, occurs at about $8.5 \mathrm{eV}$.

Information on the empty level structure, however, is scarce. The negative ion mass spectra are dominated by formation of long-lived parent molecular anions at incident electron energies of zero, 0.9 and $1.2 \mathrm{eV}^{23}$ Interestingly the molecular anions observed around $1 \mathrm{eV}$ were found $^{23}$ to possess an exceptionally long $(\sim 20 \mu \mathrm{s})$ lifetime, and were 
attributed to core-excited Feshbach resonances (electron capture accompanied by excitation of the neutral molecule). In addition, experimental data for the gas-phase adiabatic electron affinity $\left(\mathrm{EA}_{a}\right)$ of azobenzene have been reported. There is however a large discrepancy between the values of $0.62 \mathrm{eV}^{24}$ and $0.57 \mathrm{eV}^{25}$ (obtained with electrontransfer equilibria techniques) and that (approximately $1 \mathrm{eV}$ ) recently obtained ${ }^{26}$ by means of anion photoelectron spectroscopy. Similarly, the $\mathrm{EA}_{2} \mathrm{~s}$ evaluated with different theoretical methods also range from $0.57 \mathrm{eV}^{27}$ to $1.05 \mathrm{eV}^{11}$

Electron transmission spectroscopy (ETS) ${ }^{28}$ is one of the most suitable means for detecting gas-phase temporary anion states, which appear as sharp structures, "resonances", in the electron-molecule scattering cross section. Because electron attachment is rapid with respect to nuclear motion, anions are formed with the equilibrium geometry of the neutral molecule. The impact energies at which electron attachment occurs are properly denoted as vertical attachment energies (VAEs) and are the negatives of the vertical electron affinities $\left(\mathrm{EA}_{v} \mathrm{~s}\right)$.

Additional information can be supplied by dissociative electron attachment spectroscopy (DEAS) ${ }^{29}$ which measures the yield of massselected negative ions as a function of the impact electron energy. When suitable energetic conditions apply, the decay of unstable molecular anions formed by resonance attachment can follow a dissociative channel which generates a negative fragment and a neutral radical, in kinetic competition with simple re-emission of the extra electron. Measurements of anion currents, as a function of the incident electron energy, can thus reveal the formation of long-lived parent anions or dissociative decay channels of resonance processes.

In the present study, trans-azobenzene is analyzed by means of ET and DEA spectroscopies. Density functional theory (DFT) and Hartree-Fock (HF) theoretical calculations are employed to reproduce the energies of vertical electron attachment and characterize the nature of the temporary anion states observed in the ET spectra. DFT calculations are also used to describe the electronically excited states of the anion in the neutral geometry and evaluate the energy of the lowest lying core-excited resonance, and to calculate the thermodynamic energy thresholds for possible dissociative decay channels of the parent anion. 


\section{Experimental}

Our electron transmission apparatus is in the format devised by Sanche and Schulz ${ }^{28}$ and has been previously described..$^{30}$ To enhance the visibility of the sharp resonance structures, the impact energy of the electron beam is modulated with a small ac voltage, and the derivative of the electron current transmitted through the gas sample is measured directly by a synchronous lock-in amplifier. Each resonance is characterized by a minimum and a maximum in the derivative signal. The energy of the midpoint between these features is assigned to the VAE. The present spectrum has been obtained by using the apparatus in the "high-rejection" mode ${ }^{31}$ and is, therefore, related to the nearly total scattering cross section. The electron beam resolution was about $50 \mathrm{meV}$ (fwhm). The energy scale was calibrated with reference to the $\left(1 S^{1} 2 S^{2}\right)^{2} S$ anion state of He. The estimated accuracy is \pm 0.05 or $\pm 0.1 \mathrm{eV}$, depending on the number of decimal digits reported. The sample (commercially available) was heated to about 60 ${ }^{\circ} \mathrm{C}$ to attain a suitable vapor pressure.

The collision chamber of the ETS apparatus has been modified ${ }^{32}$ to allow for ion extraction at $90^{\circ}$ with respect to the electron beam direction. Ions are then accelerated and focused toward the entrance of a quadrupole mass filter. Alternatively, the total anion current can be collected and measured with a picoammeter at the walls of the collision chamber (about $0.8 \mathrm{~cm}$ from the electron beam). Measurements of the total and mass-selected anion currents were obtained with an incident electron beam current about twice as large as that used for the ET experiment. The energy spread of the electron beam increased to about $120 \mathrm{meV}$, as evaluated from the width of the $\mathrm{SF}_{6}{ }^{-}$signal at zero energy used for calibration of the energy scales.

Calculations were performed with the Gaussian o3 set of programs. ${ }^{33}$ Evaluation of the virtual orbital energies (VOEs) of the neutral molecule was performed at the B3LYP/6-31G(d) level ${ }^{34}$ and used the Dunning/Huzinaga valence double-zeta $\mathrm{D}_{55} \mathrm{~V}^{35}$ (C,O: 9s,5p/4s,2p; $\mathrm{H}: 4 s / 2 s$ ) basis sets, with the MP2/6-31G(d)-optimized geometries. The $\mathrm{EA}_{v}$ was calculated as the difference of the total energy of the neutral and the lowest anion state, both in the optimized geometry of the neutral state, using the B3LYP hybrid functional with the standard 6-31G(d) and 6-31+G(d) basis sets. The adiabatic electron affinity 
$\left(\mathrm{EA}_{\mathrm{a}}\right)$ was obtained as the energy difference between the neutral and the lowest anion state, each in its optimized geometry. Vertical excitation energies and oscillator strengths were calculated with the TDDFT method, ${ }^{36}$ employing the B3LYP functional.

\section{Results and discussion}

\section{Electron transmission spectrum and calculated VAEs}

In agreement with experimental data and previous theoretical results, ${ }^{11}$ the present B3LYP and MP2 calculations predict that in the equilibrium geometry of trans-azobenzene the two phenyl rings and the $\mathrm{N}=\mathrm{N}$ double bond are coplanar ( $C_{2 \mathrm{~h}}$ point group). Azobenzene possesses seven empty $\pi^{*}$ MOs, deriving from mixing between the $\pi^{*}$ orbitals of the phenyl and $\mathrm{N}=\mathrm{N}$ groups. The VAEs measured in the ET spectra of the reference molecules benzene ${ }^{30}\left(1.12 \mathrm{eV}, \pi^{*} \mathrm{e}_{2 \mathrm{u}} ; 4.82 \mathrm{eV}\right.$, $\left.\pi^{*} \mathrm{~b}_{2 \mathrm{~g}}\right)$ and trans-dimethyldiazene $37 \mathrm{CH}_{3} \mathrm{~N}=\mathrm{NCH}_{3}\left(0.83 \mathrm{eV}, \pi^{*}{ }_{\mathrm{NN}}\right)$ indicate that electron capture into the empty $\pi^{*}$ MOs of azobenzene is expected to occur at energies lower than $6 \mathrm{eV}$. Systematic ETS studies $^{38}$ have demonstrated that benzenoid hydrocarbons not substituted by third-row or heavier heteroatoms do not give rise to distinct lowenergy resonances associated with empty MOs of (local) $\sigma$ symmetry, so that signals associated with electron capture into $\sigma^{*}$ MOs are not expected. It should also be recalled that formation of stable anion states cannot be observed in ETS.

The ET spectrum of trans-azobenzene in the $0-6.5 \mathrm{eV}$ energy range is reported in Figure 1. Six narrow resonances are displayed at 0.88 , 1.29, 1.98, 3.1, 3.7 and $4.76 \mathrm{eV}$. Moreover, the first resonance clearly displays vibrational structures with an energy spacing of about 126 $\mathrm{meV}$, close to that measured in the $\mathrm{e}_{2 \mathrm{u}}\left(\pi^{*}\right)$ anion state of benzene. ${ }^{39}$ The widths, as given by the energy separation between minimum and maximum in the derivative signal of the ET spectrum, are relatively small (0.36, 0.32, 0.36, 0.32, 0.44 and $0.62 \mathrm{eV}$, respectively), including the two weaker features at 3.1 and $3.7 \mathrm{eV}$.

A qualitative perturbational analysis based on symmetry considerations indicates that the (antibonding) $\pi{ }^{*}$ NN orbital $\left(b_{g}\right.$ symmetry in the $C_{2 h}$ point group) of the central group cannot mix with the $\left(\mathrm{a}_{\mathrm{u}}\right)$ 


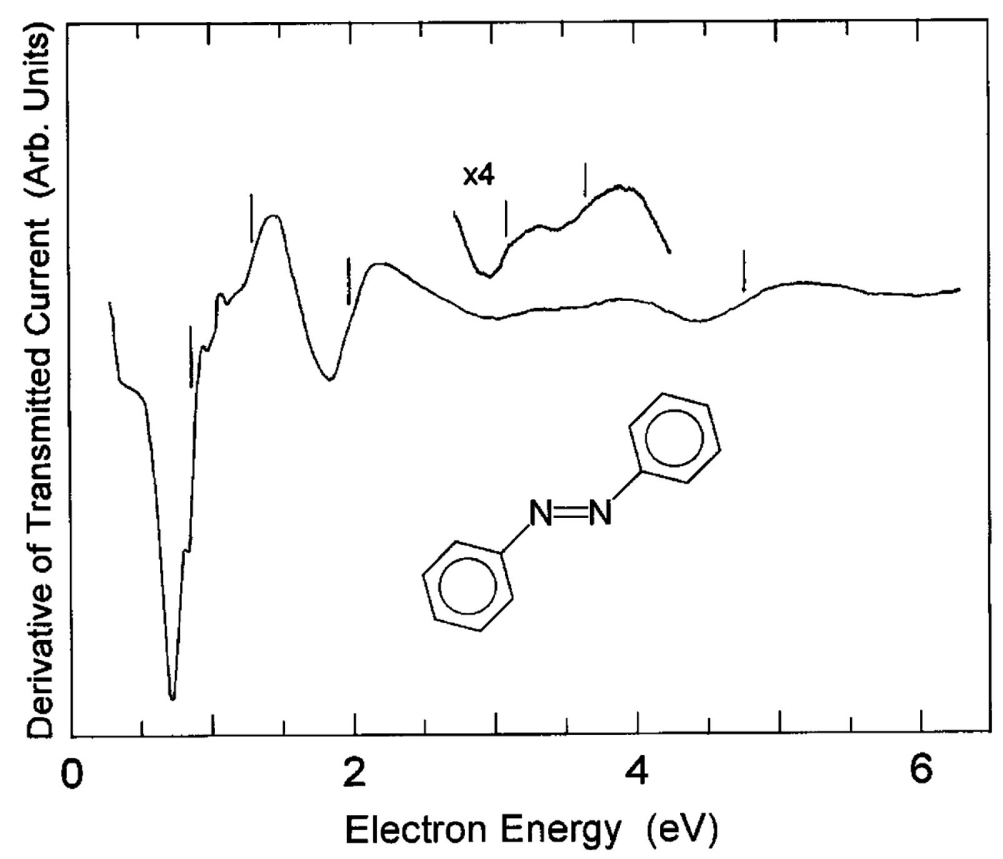

Figure 1 Derivative of transmitted current, as a function of electron energy, in gasphase trans-azobenzene. Vertical lines locate the VAEs.

in-phase combination of the symmetric components of the degenerate benzene $\mathrm{e}_{2 \mathrm{u}}\left(\pi^{*}\right)$ lowest unoccupied MO (LUMO). In addition, both the $\left(b_{g}\right)$ in-phase and $\left(a_{u}\right)$ out-of-phase combinations of the antisymmetric $\mathrm{e}_{2 \mathrm{u}}$ components, because of their nodes at the substituted carbon atoms, are not expected to interact with the $\pi^{*}{ }_{\mathrm{NN}}$ orbital regardless of their symmetry. Thus three $\pi^{*}$ anion states of azobenzene are expected to possess mainly benzene ring character, with VAEs around $1 \mathrm{eV}$ (i.e., close to the corresponding benzene VAE). At variance, the remaining $\left(b_{g}\right)$ out-of-phase combination of the symmetric benzene $\mathrm{e}_{2 \mathrm{u}}$ components will strongly mix with the central $\pi^{*}{ }_{\mathrm{NN}}$ orbital, giving rise to the LUMO of azobenzene and to a MO lying well above the $e_{2 u}$ benzene LUMO. Finally, the two totally antibonding $b_{2 g}\left(\pi^{*}\right)$ orbitals of benzene $(\mathrm{VAE}=4.82 \mathrm{eV})$, because of a large energy separation, should interact only slightly with the $\pi^{*}{ }_{\mathrm{NN}}$ orbital, so that the two corresponding MOs of azobenzene are expected to be close in energy, with a VAE of about $4.8 \mathrm{eV}$.

The above description of the empty level structure of azobenzene is summarized more quantitatively in the scheme of Figure 2, based 

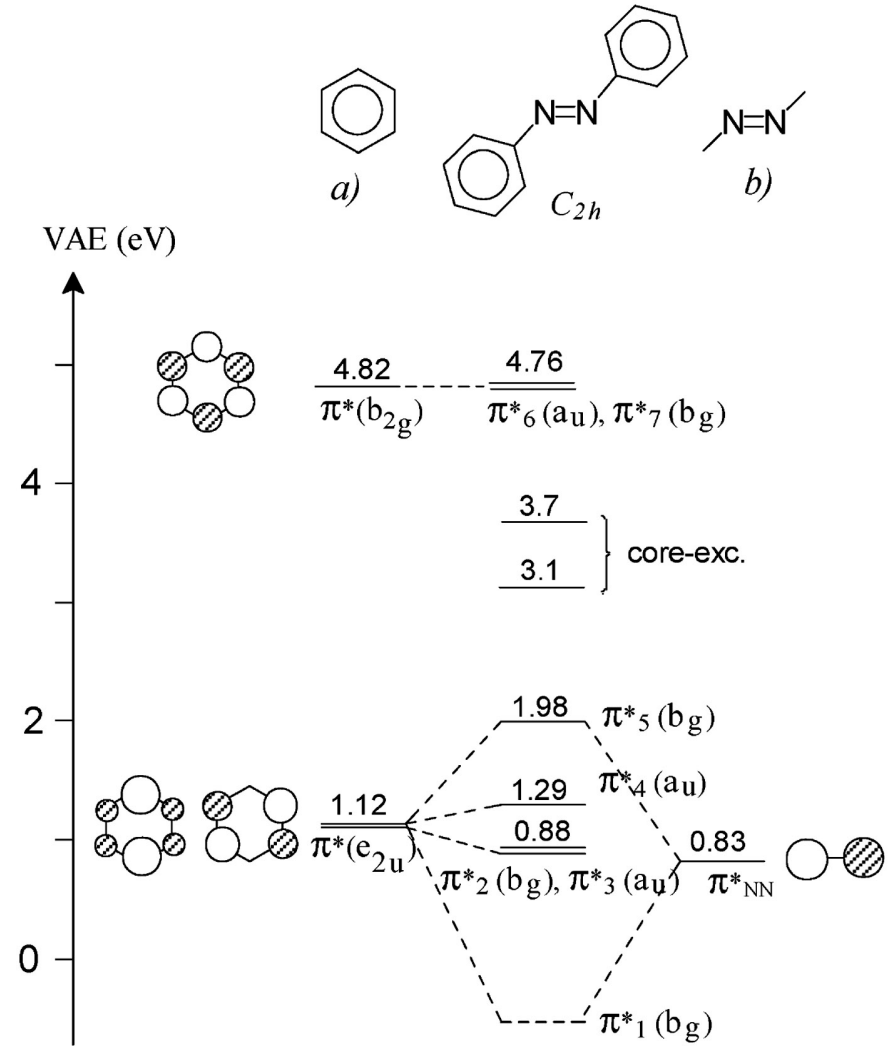

Figure 2 Correlation diagram of the VAEs (eV) measured in trans-azobenzene and the reference molecules benzene and dimethyldiazene. (a) from ref. 30; (b) from ref. 37 .

on the VAEs measured in the ET spectra of trans-azobenzene and the reference molecules benzene and dimethyldiazene. In particular, this picture highlights two points. The lowest vertical anion state of transazobenzene, not detected in the ET spectrum, is stable (i.e., positive $\mathrm{EA}_{v}$ ), and the two weak features detected at 3.1 and $3.7 \mathrm{eV}$, not accounted for by simple electron capture into empty $\pi^{*}$ MOs, must be associated with core-excited resonances.

To give further support to this interpretation of the spectral features, we have carried out theoretical calculations. An approach adequate for describing unstable anion states involves difficulties not encountered for neutral or cation states. ${ }^{40-43}$ The most correct method is, in principle, the calculation of the total scattering cross section with the use of continuum functions, but complications arise from the lack of an accurate description of the electron-molecule interaction. ${ }^{44}$ 
The VAE corresponding to the stable anion can be obtained as the difference between the energy of the anion and that of the neutral state, both at the optimized geometry of the neutral species. A proper description of the spatially diffuse electron distributions of anions normally requires a basis set with diffuse functions. ${ }^{45,46}$ However, calculated anion state energies decrease as the basis set is expanded, so the choice of an appropriate basis set is a delicate task. ${ }^{40,41,47,48}$

The Koopmans' theorem (KT) approximation ${ }^{49}$ neglects correlation and relaxation effects. However, Chen and Gallup ${ }^{42}$ and Staley and Strnad ${ }^{40}$ demonstrated the occurrence of good linear correlations between the $\pi{ }_{C=C}$ VAEs measured in a large number of alkenes and benzenoid hydrocarbons and the corresponding virtual orbital energies (VOEs) of the neutral molecules obtained with simple KT Hartree-Fock calculations, using basis sets which do not include diffuse functions. The best correlation was found using the HF/D95V//MP2/6$31 \mathrm{G}(\mathrm{d})$ method. It has recently been shown ${ }^{47}$ that the neutral state $\pi^{*}$ VOEs obtained with B3LYP/6-31G(d) calculations also supply a good linear correlation with the corresponding VAEs measured over a variety of different families of unsaturated compounds, including heterosubstituted compounds. It should be expected that a more accurate correlation would result if "training" compounds were employed that were structurally closer to the subject molecule. In the case of azobenzene, the use of the correlation derived from a study ${ }^{50}$ of $\pi^{*}$ orbitals in a series of alternating phenyl and ethynyl groups is appropriate and applied to the B3LYP/6-31G(d) VOE calculations.

Table 1 reports the $\pi *$ VOEs of trans-azobenzene obtained with HF/D95V (geometry optimized at the MP2/6-31G(d) level) and B3LYP/6-31G(d) calculations. The values scaled with the corresponding empirical linear equations $\left(\mathrm{VAE}_{(\mathrm{eV})}=0.73865 \mathrm{VOE}-1.4100^{40}\right.$ and $\mathrm{VAE}_{(\mathrm{eV})}=(\mathrm{VOE}+1.41) / 1.24,{ }^{50}$ respectively) reported in the literature are also given, together with the experimental VAEs, for the sake of comparison.

While both sets of scaled VOEs are close to each other, the best overall match to experiment is obtained with the phenyl-ethynyl scaling as expected. In particular, there is excellent agreement for the $\pi_{5}{ }^{*}$ anion, the state corresponding to occupation of the antibonding NN and ring orbital. This lends increased credibility to the value for the lowest VAE of $-0.83 \mathrm{eV}$, that is, a positive $\mathrm{EA}_{v}$ of $0.83 \mathrm{eV}$, for an anion state that is not directly detectable by ETS. According to both 
Table 1 Calculated $\pi^{*}$ VOEs (eV) of trans-azobenzene, scaled values (see text) and experimental VAEs

\begin{tabular}{|c|c|c|c|c|c|}
\hline \multirow[b]{2}{*}{ Orbital } & \multicolumn{2}{|c|}{$\mathrm{HF} / \mathrm{D} 95 \mathrm{~V} / / \mathrm{MP} 2 / 6-31 \mathrm{G}(\mathrm{d})$} & \multicolumn{2}{|c|}{ B3LYP/6-31G(d) } & \multirow[b]{2}{*}{ Expt. VAE } \\
\hline & VOE & Scaled ${ }^{a}$ & VOE & Scaled $^{b}$ & \\
\hline$\pi_{\text {ring }}^{*}\left(b_{g}\right)$ & $9 \cdot 328$ & 5.48 & 4.749 & 4.75 & \\
\hline$\pi^{*}{ }_{\text {ring }}^{*}\left(a_{\mathrm{u}}^{\mathrm{s}}\right)$ & 8.864 & 5.14 & 4.446 & 4.50 & $4 \cdot 76$ \\
\hline$\pi^{*}{ }_{N N-\pi^{*} \text { ring }}\left(b_{g}\right)$ & $5 \cdot 370$ & 2.56 & 1.284 & 1.96 & 1.98 \\
\hline$\pi^{*}{ }_{\text {ring }}\left(a_{\mathrm{u}}\right)$ & 3.763 & 1.37 & 0.273 & 1.14 & 1.29 \\
\hline$\pi^{*}{ }_{\text {ring }}\left(a_{u}\right)$ & $3 \cdot 345$ & 1.06 & -0.073 & 0.86 & \\
\hline$\pi_{\text {ring }}^{*}\left(b_{g}\right)$ & 3.261 & 1.00 & -0.100 & 0.84 & 0.88 \\
\hline$\pi^{*}{ }_{\text {NN }}+\pi^{*}{ }_{\text {ring }}\left(b_{g}\right)$ & 1.034 & -0.65 & -2.169 & -0.83 & $<0$ \\
\hline
\end{tabular}

a. Ref. 40.

b. ref. 50 .

theoretical methods, the LUMO is localized over the entire molecule, but with a predominant $\pi{ }_{\text {NN }}^{*}$ character (see Figure 3).

The second and third scaled VOEs differ only by $20 \mathrm{meV}$ while the fourth lies about $0.3 \mathrm{eV}$ higher. These three empty MOs are predicted to be essentially localized only on the benzene rings. The unresolved contributions from the first two are associated with the first resonance displayed by the ET spectrum (VAE $=0.88 \mathrm{eV})$ and the third one with the second resonance of the spectrum $(\mathrm{VAE}=1.29 \mathrm{eV})$. The next higher $\mathrm{MO}$ as noted above is the antibonding counterpart of the LUMO and gives rise to the resonance at $1.98 \mathrm{eV}$. Finally, the resonance at $4.76 \mathrm{eV}$ is ascribed to the unresolved contributions from the two combinations of the higher-lying benzene $\pi^{*}$ orbitals. We note that

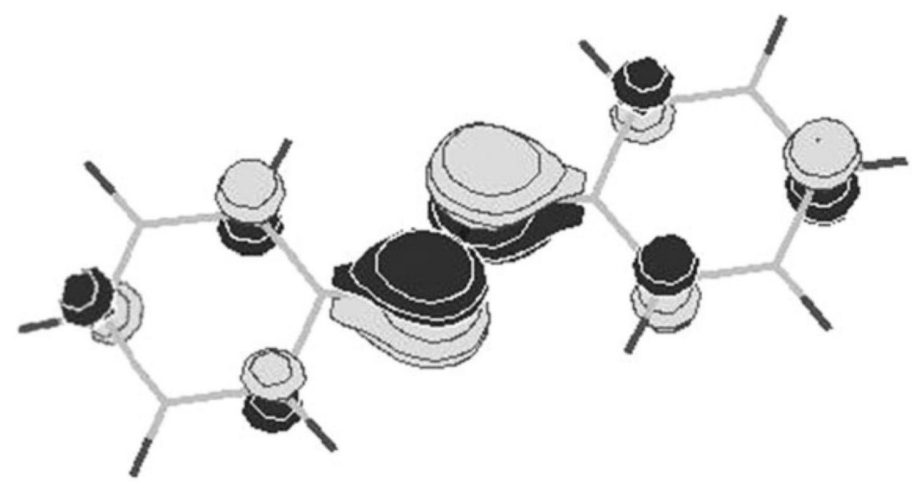

Figure 3 Representation of the LUMO of trans-azobenzene, as supplied by B3LYP/6$31 \mathrm{G}(\mathrm{d})$ calculations. 
the phenyl-ethynyl scaling gives considerably better values for these two than the scalings determined from a broader range of molecules. Overall, the scaled VOEs confirm the assignments of the ET spectral features to shape resonances, except for the two narrow but weak signals at 3.1 and $3.7 \mathrm{eV}$.

Table 2 reports the energies of the first vertical and adiabatic (geometrically relaxed) anion states relative to the neutral ground state (i.e., the negative of the $\mathrm{EA}_{v}$ and $\mathrm{EA}_{a}$, respectively) obtained with B3LYP calculations, using basis sets without diffuse functions $(6-31 G(d))$ and with the smallest addition of diffuse functions (6$31+G(d)$, with $s$ and $p$ type diffuse functions at the non hydrogen atoms). It is known (see for instance refs. 47 and 48) that the neutral/ anion energy difference obtained with the former basis set underestimates the EAs, due to its inadequacy for describing spatially diffuse anion states. On the other hand, the choice of a basis set which gives a satisfactory description of the energy and nature of the anion is a priori not obvious. ${ }^{48}$ In general, the more stable (bound) is the anion state, the smaller is the need to augment the basis set with diffuse functions. With both basis sets (see Table 2) the difference between $\mathrm{EA}_{a}$ and $\mathrm{EA}_{v}$ is relatively small $(0.22 \mathrm{eV})$, indicating a relatively small geometrical relaxation of the anion. According to the calculations, the main geometrical change is the increase (0.07 $\AA$ ) of the $\mathrm{N}-\mathrm{N}$ bond distance, in line with the $\mathrm{N}-\mathrm{N}$ antibonding character of the singly occupied MO of the anion. However, the absolute $\mathrm{EA}_{v}$ and EA values supplied by the two basis sets are sizably different (see Table 2 ), the $6-31+G(d)$ values being almost 0.5 eV larger. Intermediate values were recently found ${ }^{11}$ using a basis set $\left(6-311 G^{* *}\right)$ without

Table 2 B3LYP energies (only electronic contributions, eV) relative to the ground neutral state of trans-azobenzene

\begin{tabular}{lcc} 
Trans-azobenzene & $6-31 \mathrm{G}(\mathrm{d})$ & $6-31+\mathrm{G}(\mathrm{d})$ \\
\hline Neutral state & 0.00 & 0.00 \\
Vertical anion & -0.51 & -0.97 \\
Adiabatic anion & -0.74 & -1.19 \\
$\mathrm{C}_{6} \mathrm{H}_{5} \mathrm{NN}^{\bullet}+\mathrm{C}_{6} \mathrm{H}_{5}^{-}$ & 2.81 & 2.00 \\
$\mathrm{C}_{6} \mathrm{H}_{5}+\mathrm{N}_{2}+\mathrm{C}_{6} \mathrm{H}_{5}^{-}$ & 2.88 & 2.01 \\
$\mathrm{C}_{6} \mathrm{H}_{5} \mathrm{~N}^{\bullet}+\mathrm{C}_{6} \mathrm{H}_{5} \mathrm{~N}^{-}$ & 3.19 & 2.56 \\
{$[\mathrm{M}-\mathrm{H}]^{-}+\mathrm{H}^{\bullet}$} & $4.25 \pm 0.09$ & $3.66 \pm 0.07$
\end{tabular}

a. Average over the five positions of the $\mathrm{H}$ atoms. 
diffuse functions, but somewhat larger than the 6-31G(d) basis set. Analogously, the $6-31 \mathrm{G}(\mathrm{d})$ threshold energies for production of negative fragments are $0.6-0.8 \mathrm{eV}$ larger than those obtained with the $6-31+G(d)$ basis set (see Table 2), due to the inadequacy of the minimal basis set for describing spatially diffuse anion states.

Combining the calculated ground state anion relaxation energy of $0.22 \mathrm{eV}$ with the predicted $\mathrm{EA}_{v}$ of $0.83 \mathrm{eV}$ from our scaling procedure, we find an $\mathrm{EA}_{a}$ of $1.05 \mathrm{eV}$ which is intermediate and entirely consistent with the threshold value of $\sim 1 \mathrm{eV}$ determined by the PES experiment of Paik et al. ${ }^{26}$ and the EA a predicted by the B3LYP/6-31+G(d) calculations (1.19 eV, see Table 2).

\section{DEAS results}

Figure 4 reports the total anion current measured at the walls of the collision chamber $(0.8 \mathrm{~cm}$ from the electron beam) and the parent molecular anion $\left(\mathrm{M}^{-}, m / e=182\right)$ current selected through a mass filter, as a function of the incident electron energy. The energies of

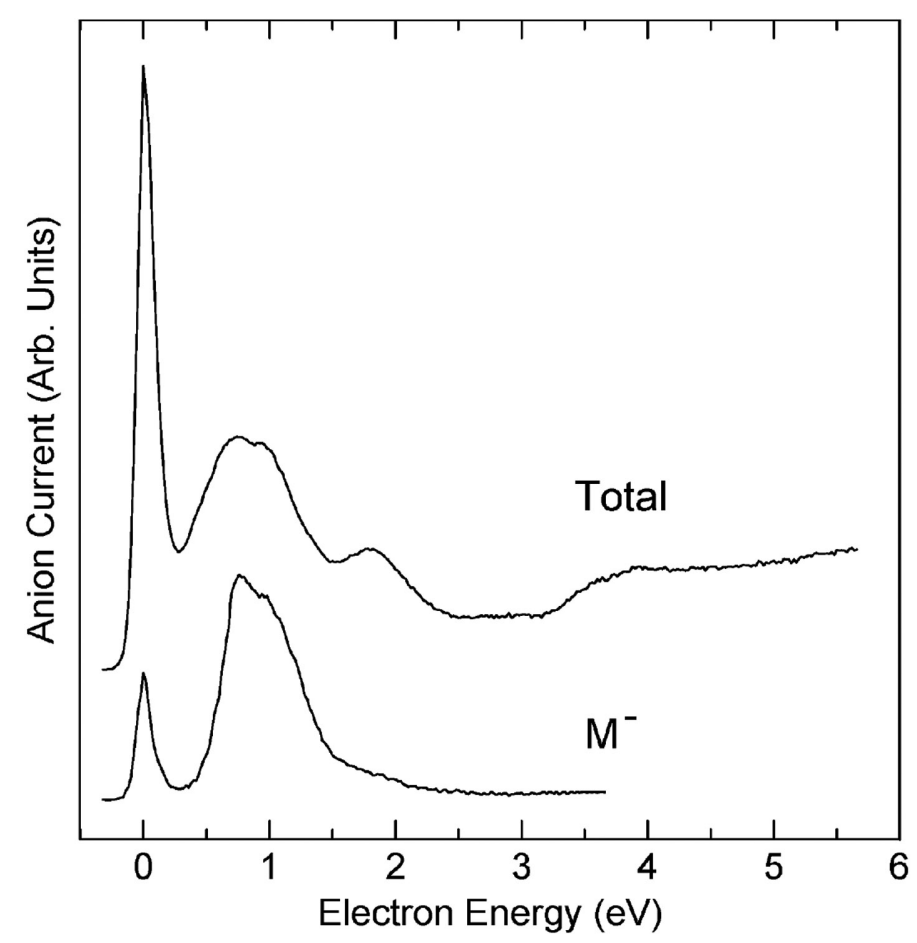

Figure 4 Total (Upper curve) and parent molecular anion (Lower curve) currents, as a function of the incident electron energy, measured in gas-phase trans-azobenzene. 
Table 3 Peak energies (eV) in the total anion current of trans-azobenzene measured at the collision chamber and the parent molecular anion current $(m / e=182)$ detected through a mass filter, and relative intensities (from peak heights)

\begin{tabular}{lllll} 
Total & & & \multicolumn{2}{l}{ Molecular anion } \\
\cline { 5 - 5 } Peak en. & Rel. int. & & Peak en. & Rel. int. \\
\hline 4.0 & 16 & & \\
$\sim 3$ & Weak & 20 & 1.8 & V. weak \\
1.8 & 38 & 1.0 & 90 \\
1.0 & 40 & 0.8 & 100 \\
0.8 & 100 & 0.0 & 57 \\
\hline 0.0 & & & \\
\hline
\end{tabular}

the maxima, together with the relative peak heights, are reported in Table 3. Both the total and the $m / e=182$ anion currents display intense peaks at zero energy, $0.8 \mathrm{eV}$ and $1.0 \mathrm{eV}$. The smaller relative intensity of the zero energy peak in the mass-selected spectrum is likely due to kinetic energy discrimination in the anion extraction efficiency. The total anion current also shows a pronounced maximum at $1.8 \mathrm{eV}$, a flat signal around $3 \mathrm{eV}$ and an additional broad peak near $4 \mathrm{eV}$, following a rise at about $3.4 \mathrm{eV}$. The latter two signals could be associated with the features observed at 3.1 and $3.7 \mathrm{eV}$ in the ET spectrum, assigned to core-excited resonances.

An evaluation of the total anion current absolute cross section can be obtained from comparison of absolute cross sections reported in the literature with our measurements on the same compounds (see ref. 51 for more details). Application of this procedure leads to an absolute cross section of the order of magnitude of $10^{-16} \mathrm{~cm}^{2}$ for the maxima around $1 \mathrm{eV}$. However, due to the relatively low volatility of azobenzene and the fact that in the apparatus employed the sample vapor pressure is measured outside the collision chamber, this value has to be considered as an upper limit.

The peak at $1.8 \mathrm{eV}$ in the total anion current has a very weak counterpart in the mass-selected $\mathrm{M}^{-}$current, indicating that the lifetime of this negative species is barely sufficient for detection through the mass filter. The mass-selected $\mathrm{M}^{-}$current thus indicates that the total anion current below $2 \mathrm{eV}$ is essentially accounted for by the parent molecular anion. 
Except for the very weak signal observed here at $1.8 \mathrm{eV}$, there is good agreement between the present data (peak energies and relative intensities) and the $\mathrm{M}^{-}$current previously reported by Vasil'ev et al. ${ }^{23}$ These authors also detected several negative fragment currents, the most intense being $[\mathrm{M}-\mathrm{H}]^{-}(1.6 \%), \mathrm{C}_{6} \mathrm{H}_{5} \mathrm{~N}^{-}(0.3 \%)$ and $\mathrm{C}_{6} \mathrm{H}_{5}-(0.16 \%)$, peaking at $6.8,5.2$ and $4.3 \mathrm{eV}$, respectively. Due to their small abundance, we did not try to reproduce these signals. However, we have calculated (see Table 2) the thermodynamic energy thresholds for the formation of these negative species. The results are consistent with their absence below $2 \mathrm{eV}$, and the calculated energy ordering of their thermodynamic thresholds parallels the ordering of the above reported experimental peak energies.

The lifetime required for observation of negative species through the mass filter of our apparatus is of the order of magnitude of $1 \mu \mathrm{s}$, whereas that required to reach the walls of the collision chamber (total anion current) is about two orders of magnitude smaller. The $\mathrm{M}^{-}$ peak at zero energy can clearly be associated with formation of vibrationally excited levels of the ground anion state, in keeping with the usual interpretation of long lived parent anions found in a number of compounds.

The observation of parent molecular anions at energies above zero is much less common. Vasil'ev et al. ${ }^{23}$ proposed that the two $\mathrm{M}^{-}$peaks around $1 \mathrm{eV}$ are associated with core-excited resonances of Feshbach type, that is, electron capture accompanied by simultaneous electron excitation and with the anion state lying below the associated excited state of the neutral. However, the present study shows that the $0.8,1.0$ and $1.8 \mathrm{eV}$ maxima in the anion current each appear to have a corresponding $\pi^{*}$ shape resonance counterpart in the ET spectrum at 0.88 , 1.29 and $1.98 \mathrm{eV}$, respectively.

To explore this in more detail, we have plotted the (negative) derivative with respect to energy of the total anion current and the $\mathrm{M}^{-}$ current to compare with the ETS signal, which is itself the derivative of the transmitted current through the cell. These are shown, displaced vertically, in Figure 5. The vertical scales for each are different. Although the current derivatives are noisy, the key features are easily related to those observed in the ETS signal. In particular, in the $\mathrm{M}^{-}$data where the energy resolution is highest, the sharp dip near $0.7 \mathrm{eV}$ is clear. Near $2 \mathrm{eV}$ where the $\mathrm{M}^{-}$signal is weak owing to 


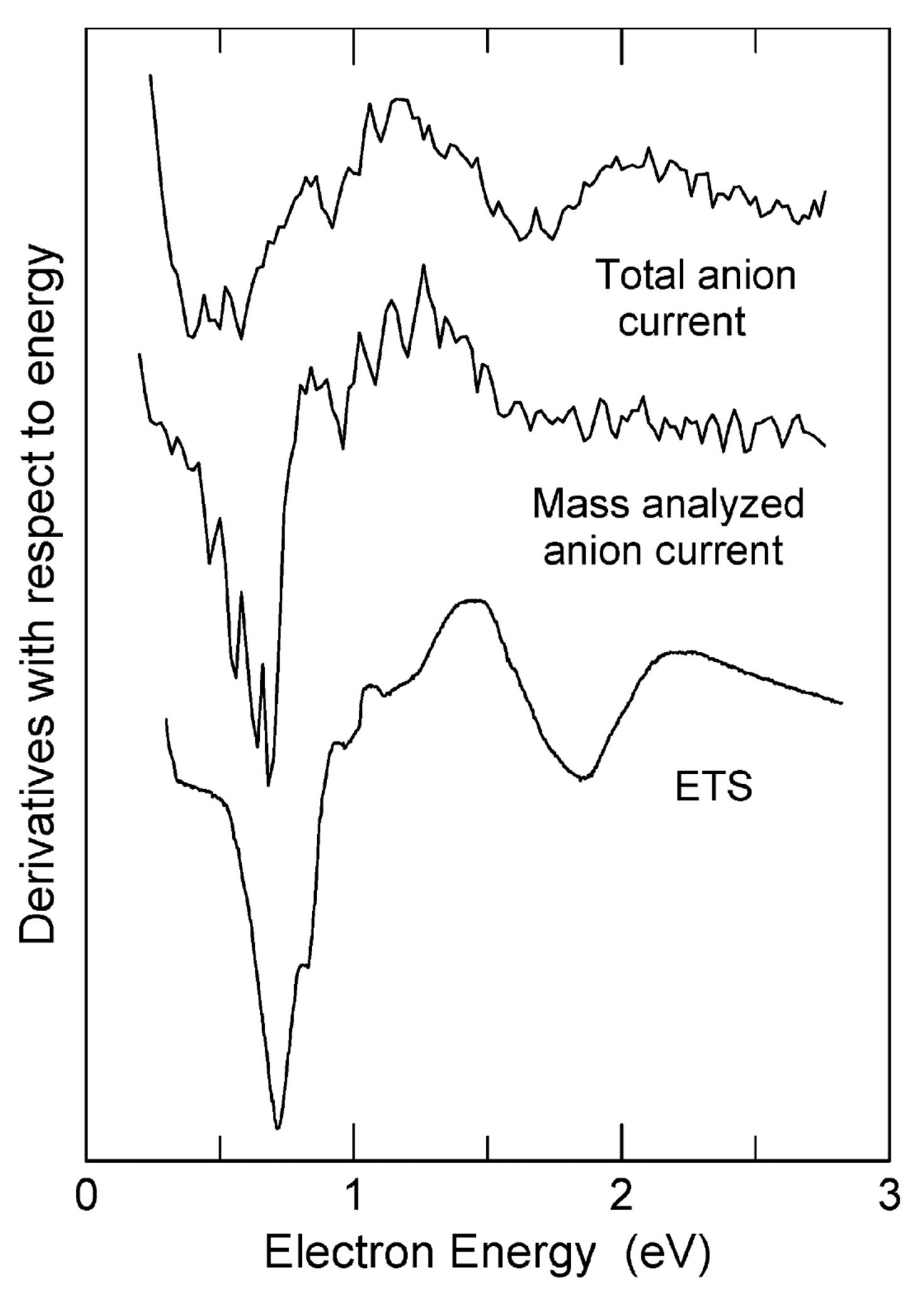

Figure 5 Derivatives with respect to energy of the total anion current, mass analyzed anion current and electron transmitted current (ETS).

the shorter lifetime of this resonance, the derivative of the total anion current matches the ETS data quite well. The ETS results appear to lie slightly higher than those from the currents but it is likely that this results from differences in energy calibration and beam resolution, although shifts owing to the shorter lifetime of resonances on their high energy side cannot be discounted.

We are not aware of examples in which such a close correspondence is evident between shape resonance profiles and energies and the peaks in the $\mathrm{M}^{-}$production. The most extensively studied system is $p$-benzoquinone for which ET spectra are available ${ }^{52,53}$ as well as measurements of parent anion yields and lifetimes. ${ }^{22,54}$ In contrast to azobenzene, only one of the three resonances observed below $3 \mathrm{eV}$ in 
ETS, the one near $2.1 \mathrm{eV}$, appears to give rise to a parent anion with long lifetime. Allan ${ }^{52}$ believes this to be a shape resonance but does not discount the possibility of an underlying core excited anion. We $\mathrm{We}^{53}$ assigned it to a core excited resonance, as the remaining features could be successfully accounted for as shape resonances. This conclusion is further supported by B3LYP/6-31G(d) scaled VOEs (not reported here) which nicely account for all spectral features except for the $2.1 \mathrm{eV}$ signal.

The mechanisms for production of metastable anions with microsecond lifetimes could include: (1) initial formation of the shape resonances followed by an internal conversion (of some or all) to highly vibrationally excited levels of the ground electronic state of the anion. This is the mechanism proposed by Cooper et al. ${ }^{54}$ and Allan ${ }^{52}$ in $p$-benzoquinone. It is perhaps surprising that in the case of azobenzene each of the shape resonances "converts" in the same way, that is, the production of long lived anions mimics the shape of the attachment cross section into the $\pi^{*}$ shape resonances. (2) Direct formation of core excited anion states which convert to long lived (microsecond) forms, perhaps as in (1) by conversion to highly excited vibrational levels of the ground anion (direct formation of a microsecond lifetime core excited state can be neglected since the structure would be too narrow to observe in the scattering cross section). (3) Direct production of the shape resonances followed by conversion to microsecond lifetime core excited anion states, if they exist. (4) Direct production of core-excited states (doublets) followed by conversion into long lived quartet states of the anion as proposed by Khvostenko et al. ${ }^{55,56}$ for pyromellitic acid imide and $p$-benzoquinone.

To investigate the energetics of core-excited resonances, we carried out TD-B3LYP calculations. The experimental transition energies ${ }^{14}$ of the two lowest singlet states $\left(\sigma-\pi^{*}\right.$ and $\left.\pi-\pi^{*}\right)$ of the neutral molecule are satisfactorily reproduced (see Table 4), in particular, the energy calculated for the $\pi-\pi^{*}$ transition is in excellent agreement with experiment.

The vertical energies of the two lowest triplet states of neutral trans-azobenzene are predicted to be significantly smaller than those of the corresponding singlet states, i.e., about $1.8 \mathrm{eV}\left(\sigma-\pi^{*}\right)$ and 2.2 $\mathrm{eV}\left(\pi-\pi^{*}\right)$ with both the $6-31 \mathrm{G}(\mathrm{d})$ and $6-31+\mathrm{G}(\mathrm{d})$ basis sets (see Table 4 ), while the energy of the adiabatic $\sigma-\pi^{*}$ transition (to give a triplet state at its optimized geometry) is predicted to be only $1.2 \mathrm{eV}$. 
Table 4 Energies (eV) of electronic transitions in gas-phase neutral and anion states of trans-azobenzene, supplied by TD-B3LYP calculations. The energies of the excited anion states relative to the ground neutral state are given in parentheses

\begin{tabular}{|c|c|c|}
\hline Excited state $^{a}$ & $6-31 G(d)$ & $6-31+G(d)$ \\
\hline & Neutral & \\
\hline$\sigma-\pi^{*} S$ vert. ${ }^{b}$ & 2.55 & 2.57 \\
\hline$\pi-\pi^{*} S$ vert. ${ }^{c}$ & 3.77 & 3.67 \\
\hline$\sigma-\pi^{*} \mathrm{~T}$ vert. & 1.78 & 1.82 \\
\hline$\pi-\pi^{*} \mathrm{~T}$ vert. & 2.19 & 2.18 \\
\hline$\sigma-\pi^{*} \mathrm{~T}$ adiab. & $\begin{array}{l}1.21 \\
\text { Vertical anion }\end{array}$ & 1.24 \\
\hline$\sigma-\pi^{*}$ D vert. & $2.10(1.27)$ & $2.12(1.29)$ \\
\hline$\pi-\pi^{*}$ D vert. & $\begin{array}{l}3.50(2.67) \\
\text { Adiabatic anion }\end{array}$ & $3.32(2.49)$ \\
\hline$\sigma-\pi^{*}$ D vert. & $1.97(0.97)$ & $2.01(1.01)$ \\
\hline$\pi-\pi^{*} D$ vert. & 2.59 (1.59) & $2.50(1.50)$ \\
\hline
\end{tabular}

a. $\mathrm{S}=$ singlet, $\mathrm{D}=$ doublet, $\mathrm{T}=$ triplet, Expt. values from ref. 14 .

b. $2.76 \mathrm{eV}$.

c. $3.78 \mathrm{eV}$.

According to the B3LYP calculations, the $\pi-\pi$ * transition from the anion state in the neutral geometry occurs in the range $3.32-3.50 \mathrm{eV}$, as given by the two basis sets. Given that the vertical anion state is predicted to be $0.83 \mathrm{eV}$ more stable than the ground neutral state, this leads to an energy of $2.49-2.67 \mathrm{eV}$ for the first $\pi-\pi^{*}$ core-excited resonance, not far from that $(3.1 \mathrm{eV})$ of the first weak resonance displayed in the ET spectrum. Similarly, the $\sigma-\pi *$ transition is predicted to lie near $1.27-1.29 \mathrm{eV}$.

We note that the $\sigma-\pi^{*}$ excited doublet anion state with the geometry optimized for the ground anion state is calculated to lie only about $1 \mathrm{eV}$ above the ground state of the neutral molecule (see Table 4), and in its own optimized geometry the excited anion could lie even lower. Notice also that either vertically or adiabatically, the $\sigma-\pi^{*}$ core-excited anion is stable with respect to the $\sigma-\pi^{*}$ (geometry optimized) triplet excited state of the neutral molecule computed to lie at $1.2 \mathrm{eV}$. Thus this anion state is a Feshbach resonance, which likely will have a longer lifetime than a shape resonance at a similar energy. Conversion of the shape resonances around $1 \mathrm{eV}$ into such a long-lived Feshbach-type core-excited anion could be possible on energetic grounds. 
The (geometry optimized) quartet anion state obtained with 3 unpaired electrons (one in each of the $\sigma, \pi{ }_{1}$ and $\pi{ }_{2}{ }_{2}$ MOs) is predicted to lie $1.9(6-31+\mathrm{G}(\mathrm{d}))$ or $2.4 \mathrm{eV}(6-31 \mathrm{G}(\mathrm{d}))$ above the ground neutral state. Thus, these results do not support the hypothesis that formation of (or conversion to) molecular anions in a quartet state may occur at an incident electron energy of $1 \mathrm{eV}$.

\section{Conclusions}

The electron transmission spectrum of trans-azobenzene displays intense resonances at $0.88,1.29,1.98$, and $4.76 \mathrm{eV}$ and two weak signals at 3.1 and $3.7 \mathrm{eV}$. The strong features are associated with vertical electron capture into empty $\pi^{*}$ MOs. Their energies are satisfactorily reproduced by calculations at the HF and B3LYP levels on the neutral molecule, once the calculated virtual orbital energies are scaled with empirical linear equations reported in the literature. This procedure also predicts the lowest vertical anion state to be $0.83 \mathrm{eV}$ more stable than the ground neutral state. The two weaker signals are ascribed to core-excited resonances. The results of TD-B3LYP calculations are consistent with the assignment of the first one to electron attachment to the $\pi^{*}$ LUMO accompanied by the lowest $\pi-\pi *$ transition.

The total anion current measured at the walls of the collision chamber shows a sharp peak at zero energy and three intense maxima at energies (o.8, 1.0 and $1.8 \mathrm{eV})$. In agreement with previously reported data, intense peaks at zero energy, 0.8 and $1.0 \mathrm{eV}$ are also observed in the parent molecular anion current $(\mathrm{m} / \mathrm{e}=182)$ detected with a mass filter, where a lifetime of the order of magnitude of $1 \mu$ s (or larger) is required.

The close similarity of the ET spectrum of trans-azobenzene with the derivatives of the molecular anion currents argues that the molecular anions observed above zero energy are associated with electron capture into empty $\pi *$ MOs. Our calculations show that these MO's have mainly benzene character. The long lifetimes of the parent molecular anions of $p$-benzoquinone and pyromellitic acid imide formed at $1.35 \mathrm{eV}$ and $1.65 \mathrm{eV}$, respectively, were explained ${ }^{55,56}$ by invoking a change of multiplicity (conversion from the initial doublet anion state to a quartet state). The present B3LYP calculations, however, do not support the occurrence of such a mechanism in trans-azobenzene at energies as low as $1 \mathrm{eV}$. 
To our knowledge, the means by which $\pi^{*}$ resonances could yield parent anions with microsecond lifetimes has not been treated in any quantitative sense. A useful connection may exist in the observation by Allan ${ }^{52}$ that shape resonances in complex molecules decay in part to high vibrational levels of the neutral molecule that are coincident with the electron energy forming the shape resonance. Such "unspecific" vibrational excitation, as Allan labels it, produces an outgoing electron energy distribution peaking near zero energy. A model study by Gauyacq ${ }^{57}$ relates this to the high density of vibrational levels of the neutral molecule at the energies of the shape resonances. In a similar manner, shape resonances could decay into the even greater density of vibrational levels of the stable anion in azobenzene which provides a virtual continuum. Since a variety of vibrational symmetries may be present, shape resonances of different symmetries will still be vibronically coupled to the continuum of the ground state of the anion. Because of the large internal energy of the anion, it is likely that the major portion of the vibrational motion occurs on a potential surface lying below that of the neutral. Indeed, this would be necessary to prevent rapid autodetachment.

A similar scenario could also be invoked for conversion of the shape resonances to nearby core-excited states of the anion. To achieve lifetimes of microseconds, it would again be necessary that these anions move to nuclear geometries where autodetachment is forbidden. It should be noted that the density of vibrational levels of the core excited anions adjacent to the shape resonance energies will be much less than that of the ground anion state, which may make this conversion less likely.

Acknowledgments Thanks are due to the Italian Ministero dell'Istruzione, dell' Università e della Ricerca for financial support. PDB acknowledges many useful conversations with G. A. Gallup.

\section{References}

1 G. S. Hartley, Nature, 1937, 140, 281.

2 N. Tamai and H. Miyasaka, Chem. Rev., 2000, 100, 1875.

3 T. Saito and T. Kobayashi, J. Phys. Chem. A, 2002, 106, 9436. 
4 T. Hugel, N. B. Holland, A. Cattani, L. Moroder, M. Seitz and H. E. Gaub, Science, 2002, 296, 1103.

5 H. Fliegl, A. Köhn, C. Hättig and R. Ahlrichs, J. Am. Chem. Soc., 2003, 125, 9821.

6 E. Wei-Guang Diau, J. Phys. Chem. A, 2004, 108, 950.

7 L. Gagliardi, G. Orlandi, F. Bernardi, A. Cembran and M. Garavelli, Theor. Chem. Acc., 2004, 111, 363.

8 H. Satzger, C. Root and M. Braun, J. Phys. Chem. A, 2004, 108, 6265.

9 C. Zhang, M.-H. Du, H.-P. Cheng, X.-G. Zhang, A. E. Roitberg and J. L. Krause, Phys. Rev. Lett., 2004, 92, 158301.

10 A. Toniolo, C. Ciminelli, M. Persico and T. J. Martınez, J. Chem. Phys., 2005, 123, 234308.

11 G. Füchsel, T. Klamroth, J. Dokić and P. Saalfrank, J. Phys. Chem. B, 2006, 110, 16337.

12 N. Henningsen, K. J. Franke, I. F. Torrente, G. Schulze, B. Priewisch, K. RuckBraun, J. Dokić, T. Klamroth, P. Saalfrank and J. I. Pascual, J. Phys. Chem. C, 2007, 111, 14843.

13 K. G. Yager and C. J. Barrett, Can. J. Photochem. \& Photobiol. A: Chemistry, 2006, 182, 250.

14 A. Biancalana, E. Campani, G. Di Domenico, G. Gorini, A. Iacoponi and G. Masetti, Spectrochim. Acta, Part A, 1999, 55, 2883.

15 N. Biswas and S. Umapathy, Chem. Phys. Lett., 1995, 236, 24.

16 I. Mita, K. Horie and K. Hirao, Macromolecules, 1989, 22, 558.

17 M. J. Comstock, J. Chi, A. Kirakosian and M. F. Crommie, Phys. Rev. B, 2005, 72, 153414 .

18 N. Tamai and H. Miyasaka, Chem. Rev., 2000, 100, 1875.

19 S. Wagner, F. Leyssner, C. Kördel, S. Zarwell, R. Schmidt, M. Weinelt, K. RückBraun, M. Wolf and P. Tegeder, Phys. Chem. Chem. Phys., 2009, DOI: 10.1039/ b823330f, in press.

20 S. Hagen, P. Kate, F. Leyssner, D. Nandi, M. Wolf and P. Tegeder, J. Chem. Phys., 2008, 129, 164102-164102.

21 T. Kobayashi, K. Yokota and S. Nagakura, J. Electron Spectrosc. Relat. Phenom., 1975, 6, 167.

22 N. E. Petrachenko, V. I. Vovna and G. G. Furin, J. Fluorine Chem., 1993, 63, 85.

23 Y. V. Vasil'ev, V. A. Mazunov and E. R. Nazirov, Org. Mass Spectrom., 1991, 26, 739 .

24 G. Briegleb, Angew. Chem., Int. Ed. Engl., 1964, 3, 617.

25 S. Ingemann, R. H. Fokkens and N. M. M. Nibbering, J. Org. Chem., 1991, 56, 607.

26 D. H. Paik, J. S. Baskin, N. J. Kim and A. H. Zewail, J. Chem. Phys., 2006, 125, 133408.

27 H. P. Gunawardena, M. He, P. A. Chrisman, S. J. Pitteri, J. M. Hogan, B. D. M. Hodges and S. A. McLuckey, J. Am. Chem. Soc., 2005, 127, 12627. 
28 L. Sanche and G. J. Schulz, Phys. Rev. A, 1972, 5, 1672.

29 G. J. Schulz, Rev. Mod. Phys., 1973, 45, 378-423.

30 A. Modelli, D. Jones and G. Distefano, Chem. Phys. Lett., 1982, 86, 434.

31 A. R. Johnston and P. D. Burrow, J. Electron Spectrosc. Relat. Phenom., 1982, 25, 119.

32 A. Modelli, A. Foffani, F. Scagnolari and D. Jones, Chem. Phys. Lett., 1989, 163, 269.

33 M. J. Frisch, G. W. Trucks, H. B. Schlegel, G. E. Scuseria, M. A. Robb, J. R. Cheeseman, V. G. Zakrzewski, J. A. Montgomery Jr, R. E. Stratmann, J. C. Burant, S. Dapprich, J. M. Millam, A. D. Daniels, K. N. Kudin, M. C. Strain, O. Farkas, J. Tomasi, V. Barone, M. Cossi, R. Cammi, B. Mennucci, C. Pomelli, C. Adamo, S. Clifford, J. Ochterski, G. A. Petersson, P. Y. Ayala, Q. Cui, K. Morokuma, D. K. Malick, A. D. Rabuck, K. Raghavachari, J. B. Foresman, J. Cioslowski, J. V. Ortiz, B. B. Stefanov, G. Liu, A. Liashenko, P. Piskorz, I. Komaromi, R. Gomperts, R. L. Martin, D. J. Fox, T. Keith, M. A. Al-Laham, C. Y. Peng, A. Nanayakkara, C. Gonzalez, M. Challacombe, P. M. W. Gill, B. Johnson, W. Chen, M. W. Wong, J. L. Andres, C. Gonzalez, M. E. Head-Gordon, S. Replogle and J. A. Pople, GAUSSIAN o3 (Revision B.05), Gaussian, Inc., Pittsburgh, PA, 2003.

34 A. D. Becke, J. Chem. Phys., 1993, 98, 5648.

35 T. H. Dunning, Jr and P. J. Hay, in Modern Theoretical Chemistry, ed. H. F. Schaefer III, Plenum, New York, 1976, pp. 1-28.

36 R. E. Stratmann, G. E. Scuseria and M. J. Frisch, J. Chem. Phys., 1998, 109, 8218.

37 A. Modelli, F. Scagnolari, D. Jones and G. Distefano, J. Phys. Chem. A, 1998, 102, 9675 .

38 A. Modelli, Trends in Chemical Physics, 1997, 6, 57.

39 I. Nenner and G. J. Schulz, J. Chem. Phys., 1975, 62, 1747.

40 S. S. Staley and J. T. Strnad, J. Phys. Chem., 1994, 98, 161.

41 M. Guerra, Chem. Phys. Lett., 1990, 167, 315.

42 D. A. Chen and G. A. Gallup, J. Chem. Phys., 1990, 93, 8893.

43 J. Simons and K. D. Jordan, Chem. Rev., 1987, 87, 535.

44 N. F. Lane, Rev. Mod. Phys., 1980, 52, 29.

45 W. J. Hehre, L. Radom, P. v. R. Schleyer and J. A. Pople, Ab initio Molecular Orbital Theory, Wiley, New York, 1986.

46 T. H. Dunning, Jr, K. A. Peterson and D. E. Woon, Basis Sets Correlation

Consistent Sets: in the Encyclopedia of Computational Chemistry, ed. P. v. R. Schleyer, John Wiley, Chichester, 1998.

47 A. Modelli, Phys. Chem. Chem. Phys., 2003, 5, 2923.

48 A. Modelli, B. Hajgató, F. J. Nixon and L. Nyulászi, J. Phys. Chem. A, 2004, 108, 7440.

49 T. Koopmans, Physica A, 1934, 1, 104. 
50 A. M. Scheer and P. D. Burrow, J. Phys. Chem. B, 2006, 110, 17751. Correction: A.M. Scheer and P. D. Burrow, J. Phys. Chem. B, 2008, 112, 10382.

51 A. Modelli, J. Phys. Chem. A, 2005, 109, 6193.

52 M. Allan, Chem. Phys., 1983, 81, 235.

53 A. Modelli and P. D. Burrow, J. Phys. Chem., 1984, 88, 3550.

54 C. D. Cooper, W. T. Naff and R. N. Compton, J. Chem. Phys., 1975, 63, 2752 (and references therein).

55 O. G. Khvostenko and G. M. Tuimedov, Rapid Commun. Mass Spectrom., 2006, 20, 3699.

56 O. G. Khvostenko, G. M. Tuimedov and U. M. Dzhemilev, Dokl. Phys. Chem., 2007, 414, 162.

57 J. P. Gauyacq, J. Phys. B, 1990, 23, 3041. 